\title{
Micro-Calcification Classification Analysis in Mammogram Images With aid of Hybrid Technique Analysis
}

\section{M.C. Shanker ( $\sim$ shankermcece@gmail.com )}

Sathyabama Institute of Science and Technology

\section{Vadivel}

Vidyajothi Institute of Technology

\section{Research Article}

Keywords: breast cancer classification, deep belief neural network, pre-processing, feature extraction, deep learning method, and chimp optimization algorithm.

Posted Date: August 9th, 2021

DOI: https://doi.org/10.21203/rs.3.rs-735873/v1

License: (c) (i) This work is licensed under a Creative Commons Attribution 4.0 International License.

Read Full License 


\title{
Micro-Calcification Classification Analysis in Mammogram Images with aid of Hybrid Technique Analysis
}

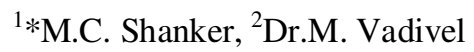 \\ ${ }^{1 *}$ Research Scholar, Department of Electronics and Communications Engineering, \\ Sathyabama Institute of Science and Technology, Chennai, India. \\ shankermcece@gmail.com \\ ${ }^{2}$ Professor, Department of Electronics and Communications Engineering, \\ Vidyajothi Institute of Technology, Hydrabad, AndraPradesh, India.
}

\begin{abstract}
The main cause of death in women is breast cancer. Early identification can contribute significantly to improving the survival rate. For diagnosis and accurate therapy automatic detection of micro-calcification is therefore essential. In the paper, an automated technique is utilized in the mammogram images according to their micro-calcification classification. The automated technique is working with the combination of Deep Belief Neural Network (DBNN) and Chimp Optimization Algorithm (COA). The proposed method is working with three phases such as pre-processing phase, feature extraction, and classification phase. In the pre-processing phase, a median filter is utilized to remove unwanted information from the images. In the feature extraction phase, Gray Level CoOccurrence Matrix (GLCM), Scale-Invariant Feature Transform (SIFT), and Hu moments are utilized to extract essential features from the mammogram images. After that, the detection and classification are performed on the mammogram images according to their micro-calcifications with the utilization of the proposed advanced deep learning method. From the classification stage, the normal and abnormal images are identified from the images. The proposed method is implemented in the MATLAB platform and analyzed their statistical performances like accuracy, sensitivity, specificity, precision, recall, and F-measure. To evaluate the effectiveness of the proposed method this is compared with the existing method such as Support Vector Machine (SVM), Random Forest (RF), and Artificial Neural Network (ANN).
\end{abstract}

Keywords: breast cancer classification, deep belief neural network, pre-processing, feature extraction, deep learning method, and chimp optimization algorithm. 


\section{Introduction}

Women on earth suffer from breast cancer as the most malignant disease. It begins because of the uncontrolled cell division, resulting in breast development of a tumor [1]. Breast cancer of malignant growths of the chest include anomalies, changes in the skin tone of the chest, a change in the fit of a fiddle, and the arrangement of a breast [2]. In general, the chest is decompressed by imaging techniques such as ultrasound, rays and attractive imagery. However, mammography is the best way to detect malignancy in the early breast that uses the lower section of the X-rays for image development. Abnormalities [3, 4] such as calcification and masses and other vague symptoms such as engineering degeneration and bilateral imbalance can be differentiated using mammography. Anomalies in which a mass, thickness, bump, or deformity is expected at the mammogram address. However, not all abuse is destructive. For example, a smooth and clear-cut line (irregularity) is generally compatible [5].

Then again, with a starburst appearance (in theory) there may be a diffuse line (protrusion) of cancer, to confirm it, a biopsy is required [6]. Microcalcifications are a small arrangement of calcium groups that may be compatible, ambiguous, or suspicious. Often these miniature calculation groups are generous [7]. Nevertheless, in one area of events, these miniature calculations may be shown in a specific category of clusters and examples. All in all, they can prove premature cells or address the underlying stage of breast disease, which can be confirmed by biopsy [8]. Radiologists divide these pictures in order to analyze breast malignancy. However, radiologists' assessment of the presence of breast disease may not be reliable because of differences in their past information and appointments [9]. Therefore, a structure of the AI-based location for breasts malignant can be used to build an insurance for the radiologist and used to diagnose breast cancer for secondary purposes [10].

In the past 20 years, AI calculations have gained prominence by observing complex questions like masonry, advancement and compilation. In the case of various image recognition issues AI (ML) based strategies have shown excellent performance [11]. There are unique numerical models based on the complexity, control information, and natural representation for the regulation and prognosis of glucose-insulin structure [12]. Previously, it helped to effectively prepare multiple ML-based PC demonstration frameworks for the problem of diabetic retinopathy. In such a way, SVM is often used for group problems as a result of its solid separation capability. To identify the malignant growth of the chest in its early stages, studies are constantly developing an intriguing AIbased structure $[13,14]$. A significant difference in these works is the use of various component extraction systems, mammogram datasets, and AI modeling. The problem of breast distinguishing can be seen as another balanced 
group issues. To begin with, mammograms can be ordered casually or strangely. While this is a strangely recognized opportunity, it is additionally labeled as kind and vicious. Most of the work in the past routinely uses models that depend on intelligence, surface, or identity manipulation [15]. In recent years, the optimization-based ML is utilized to enhance the performance of the ML in breast cancer classification from mammogram images.

The remaining part of the paper is organized as follows, Section 2 describes in detail related work analysis and Section 3 provides a comprehensive description of the proposed methodology. Section 4 presents the performance assessment of the proposed method. Section 5 presents the conclusion part of the paper.

\section{Related works}

Many different methods are available to classify breast cancer with machine learning from mammogram images. Some of the works are reviewed in this section.

Naveed Chouhan et al. [16] developed a DFeBCD method to classify mammograms as normal or abnormal based on various characteristics. There are four sets of unique characteristics. Among these are standard features based on classification codes, statistical measurements and local binary formats. With a deep convolutionary neural network (CNN), a highway network will be used to change the fourth set of features of the proposed DFeBCD Mammography.

The Advanced Densnet neural network model is used by Hua Li et al. [17], which is used to classify benign or malignant scans effectively and accurately. Initially, the mammogram image is pre-processed and the normalization prevents interference with light and can prevent excessive compatibility with smaller data sets by accepting enhancement data. In addition, insert preprocessed mammogram data into a neural network model for Alexnet, VGGNet, GoogleNet, DensNet and DSNET-II, and analysis and comparison of test results.

S. Akila Agnes et al. [18] have a fully conventional MA-CNN (Multi-scale Neural Network) to assist radiologists in diagnosing breast cancer. Mammogram images can be precisely classified as a modifiable, neural network-based method. Since the classification of feature study is related to performance tasks, transférable neural networks are ideal for extracting features for specific tasks. The proposed procedure automatically categorizes mammograms into normal, malignant and benign categories in the micro MIAS database. The model improves the accuracy of the classification system by using multi-scale filters to integrate a wider range of information contexts without negotiating the calculation speed. 
Ragab Dina A. et al. [19]. Provides a DL technology CAD method for radiologists to mammograft breast cancer lesions based on extraction and classification features. To determine the best method, four various experiments were conducted. Lastly, main analysis of components (PCA) is introduced to minimize the vector of large aspect produced by the combination of aspects and reduce computational costs.

U. Raghavendra et al. [20] have used digital mammograms to automatically classify breast cancer, including natural, benign and malignant breast cancer. In order to remove features from the proposed method, Capor uses Local Sensitivity Discrimination Analysis (LSDA). Use the lowest possible performance of classifiers to choose between AdaBoost and Fuzzy Sugeno (FSC), SVM, Probabilistic Neural Network (PNN), Naive Bayes Classifier (NBC), the k-nearest neighbor, QDA, linear discrimination analysis (Both LDA), and the Decision Treaties (DT).

\section{Proposed System Model}

Breast cancer has been the most frequent occurrence of aggressive cancer among women, which is reduced by early detection. However, due to the size and appearance of breast cancer, it is challenging to identify their cancer features such as micro-calcifications from human mammograms. Therefore, the automatic detection of micro-calcification is essential for diagnosis and accurate treatment. In this paper, ADBNN is utilized to identify breast cancer from mammogram images. This proposed approach process is illustrated in figure 1. 


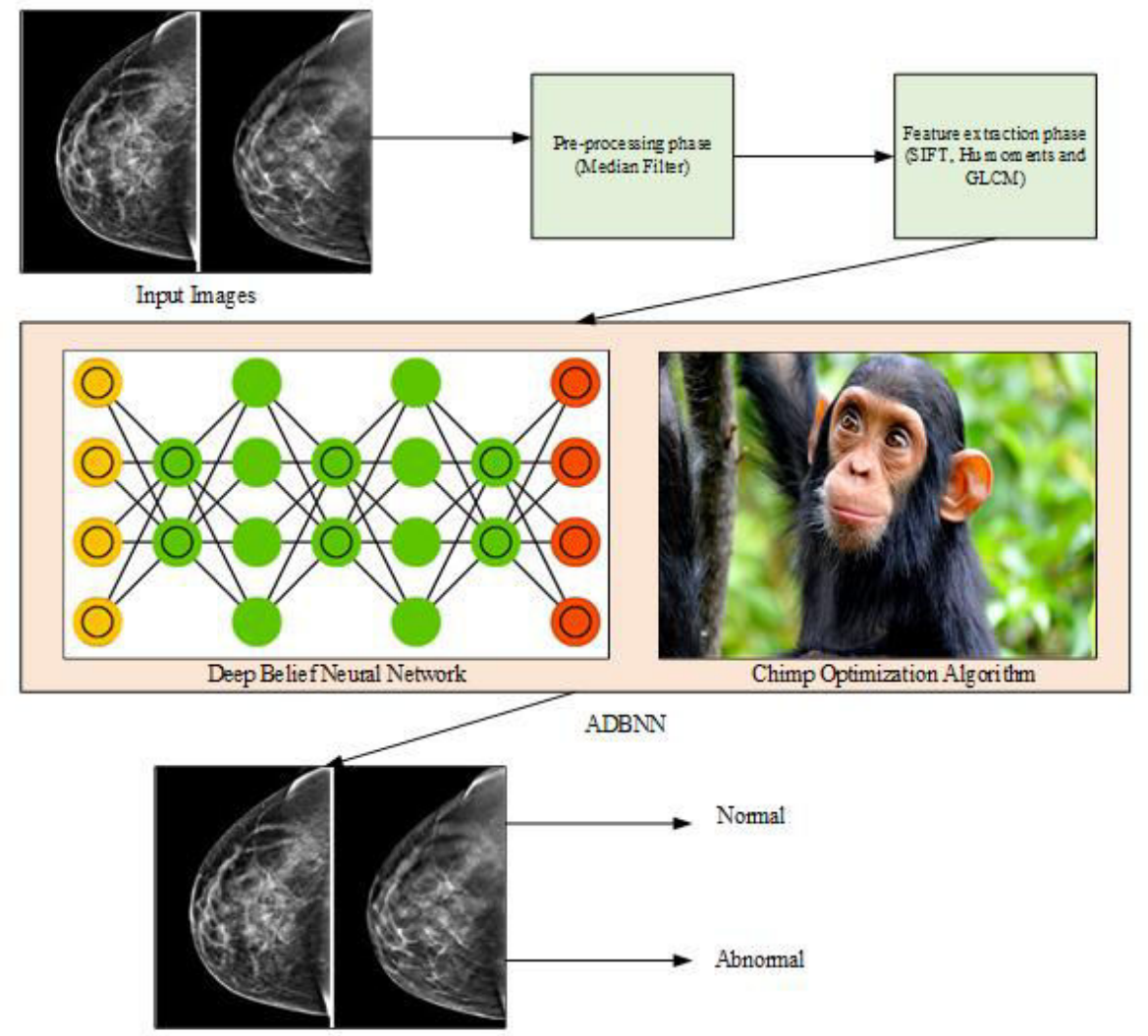

Figure. 1 Proposed Architecture

Initially, the mammogram images are collected from the open-source system. The proposed methodology is processing by three phases such as pre-processing phase, feature extraction phase, and classification phase. In the pre-processing phase, the median filter is utilized to remove unwanted noise from the input images. In the feature extraction phase, SIFT, GLCM, and hu moments features are utilized to extract essential texture and shape features. After that, the extracted features are utilized to classify breast cancer from the mammogram images. A detailed description of the proposed methodology is explained below section.

\subsection{Pre-Processing Step}

The pre-processing stage is essential to remove unwanted noise presented in collected images. In the proposed methodology, the median filter is utilized. the median filter replaces the pixel parameter in the image through the median of the filtering window. In the median filter, the closest pixel values are considered [21]. The mathematical formulation of the median filter is presented below,

$$
m f=\text { Median }(P A)=\frac{\sum_{l=1}^{N} P^{I}(N+1)}{2} \quad \text { if } N \text { is odd }
$$




$$
m f=\operatorname{Median}(P A)=\sum_{I=1}^{N} P^{I}\left(\frac{N}{2}\right)+1, \text { if } N \text { is even }
$$

Where the pixel values can be denoted as $P^{I}$. This median filter is changing the central or median pixel of the processing window. The processing pixel can be 0,255 , or different digital among 0 and 255 . This pixel exchange can be proceeding with equally in the median filter. Based on this method, the unwanted noises are removed by exchanging the pixel values.

\subsection{Feature Extraction Step}

Once complete pre-processing stage, the feature extraction is considered to extract essential features from the input images. In this proposed methodology, two types of features are considered such as GLCM, SIFT, and Hu moments.

\section{GLCM}

In this proposed methodology, the texture-based feature extraction method is selected to extract the essential features from the images such as GLCM. This feature extraction method is initially developed by Haralick and is the most important feature extraction method to extracts the texture features from the images [22]. The GLCM features behavior second-order statistics of an image through computing how often pairs of pixels with specified spatial relationships presented in an image. The GLCM matrix is consists of conditional joint probabilities of complete pair-wise combinations of gray levels presented in the particular distance and displacement distance. The displacement distance can be defined as interpixel distance. The probability condition of GLCM is presented below,

$$
P^{R}(X)=\left\{C^{I J} \mid(D, \theta)\right\}
$$

Where displacement distance can be denoted as $(D)$, particular orientation $(\theta)$ and $C^{I J}$ can be represented as a grayscale occurrence which formulated as follows,

$$
C^{I J}=\frac{P^{I J}}{\sum_{I, J=1}^{G} P^{I J}}
$$

Where, $P^{I J}$ can be described as probability value of GLCM parameters, $G$ can be represented as several gray levels.

The features are created with the computation of features for every one of the co-occurrence matrices achieved by the way of $135^{\circ}, 90^{\circ}, 45^{0}$, and $0^{\circ}$. After that, the four values are averages. Based on GLCM features, the different texture features are extracted such as Homogeneity, energy, correlation, and contrast. In this proposed 
methodology, these four features are considered from the second-order statistics. Additionally, the displacement distance and orientation process are presented below,

Kernel $=[01 ; 02 ; 03 ; 04 ; \ldots-11 ;-22 ;-33 ;-44 ; \ldots-10 ;-20 ;-30 ;-40 ; \ldots-1-1 ;-2-2 ;-3-3 ;-4-4$;

Based on kernel function, the GLCM considered four displacement distances $D=1,2,3,4$ and for angles, orientations. From the consideration of GLCM, the essential features are computed.

\section{SIFT}

The SIFT feature extraction contains the four processes such as local image descriptor, orientation assignment, optimal key point localization, and scale-space extrema detection. Initially, the scale-space extrema detection identified the key point candidates [23]. The initial step is to convolute the images with a Gaussian filter which presented below,

$$
S^{K}=S(K, Y, K \sigma)=g f(X, Y, k \sigma) * S^{K-L}
$$

Where, $S^{0}=I(X, Y), S^{K-1}=I(X, Y), K=1$ which is the input image and, Gaussian filter is formulated as follows,

$$
g f(X, Y, k \sigma)=\left(\frac{1}{2} \pi(k \sigma)^{2}\right) e^{-\frac{\left(X^{2}+Y^{2}\right)}{2 \pi(k \sigma)^{2}}}
$$

After that, the key points are localized by avoiding some low-key points in the key point localization with edge detection test and low contrast. The low contrast test avoids the unstable extrema location which presented as,

$$
X^{\prime}=-\left(\frac{\partial^{2} D O G^{-1}}{\partial X^{2}}\right)\left(\frac{\partial D O G}{\partial X}\right)
$$

Based on the SIFT, the features are extracted from the images. The extracted images are utilized to classify breast cancer from the image database.

\section{Hu moments}

The hu moments are also utilized to extract the features from the image. the Hu moments [24] use the central moments where it can be generally similar to the moments just expressed except that the values of $Y$ and $X$ in the formulations which replace through the mean values. The hu moments are mathematically presented as follows,

$$
\mu^{P, Q}=\sum_{I=0}^{N} I(X, Y)\left(X-X^{a v g}\right)^{P}\left(Y-Y^{a v g}\right)^{Q}
$$

Where,

$$
\begin{aligned}
& Y^{\text {avg }}=\frac{M 01}{M 00} \\
& X^{\text {avg }}=\frac{M 10}{M 00}
\end{aligned}
$$


The main objective of that, by mixing the various normalized central moments which is possible to generate invariant functions mentioning various characteristics of the images.

\section{ADBNN with chimp Optimization Algorithm}

The features are finally sent to the classification stage. The classification stage is utilized to identify breast cancer based on mammogram images. The proposed approach is utilized to select the emotions from the images. The hybrid ADBNN-COA is utilized and it is a combination of DBNN and COA. In the DBNN, the weight parameter is selected with the help of the COA algorithm. A detailed description of the DBNN and COA is presented below section.

\subsection{Deep Belief Neural Network}

Deeper constructions are quicker and more efficient than superficial ones. For example, a few capabilities can be summarized with a deep NN, while a system with a deep 1 requires a large number of computational units and boundaries. Superficial designs may require different components and thus, models are made to handle completely different capabilities. Nevertheless, the test for producing deeply different NNs has been slippery for some time. It develops in a way that is not straight on many levels, based on the impossibility of development techniques that rely on conventional inclinations. This has changed with the advancement of DBNNs that have been used effectively for some, including regulation, regeneration [25], scale reduction, division, data recovery, language preparation, machine technology, discourse, sound, and hybrid separation. Performance Directions. The structure of DBNNs is stabilized by a number of layers that are stacked one on top of the other so that building a system can capture the essential standards and types directly from the basic source information. Each RBM, within a certain layer, receives the contributions of the last layer and feeds them to the next layer, which gradually elevates everything individually, allowing for optimal conditions. Creating a DBNN produces each RPM independently, starting at the basic level and progressing bit by bit, with the top layer RPM. The DBNN configuration is given in Figure 2. 


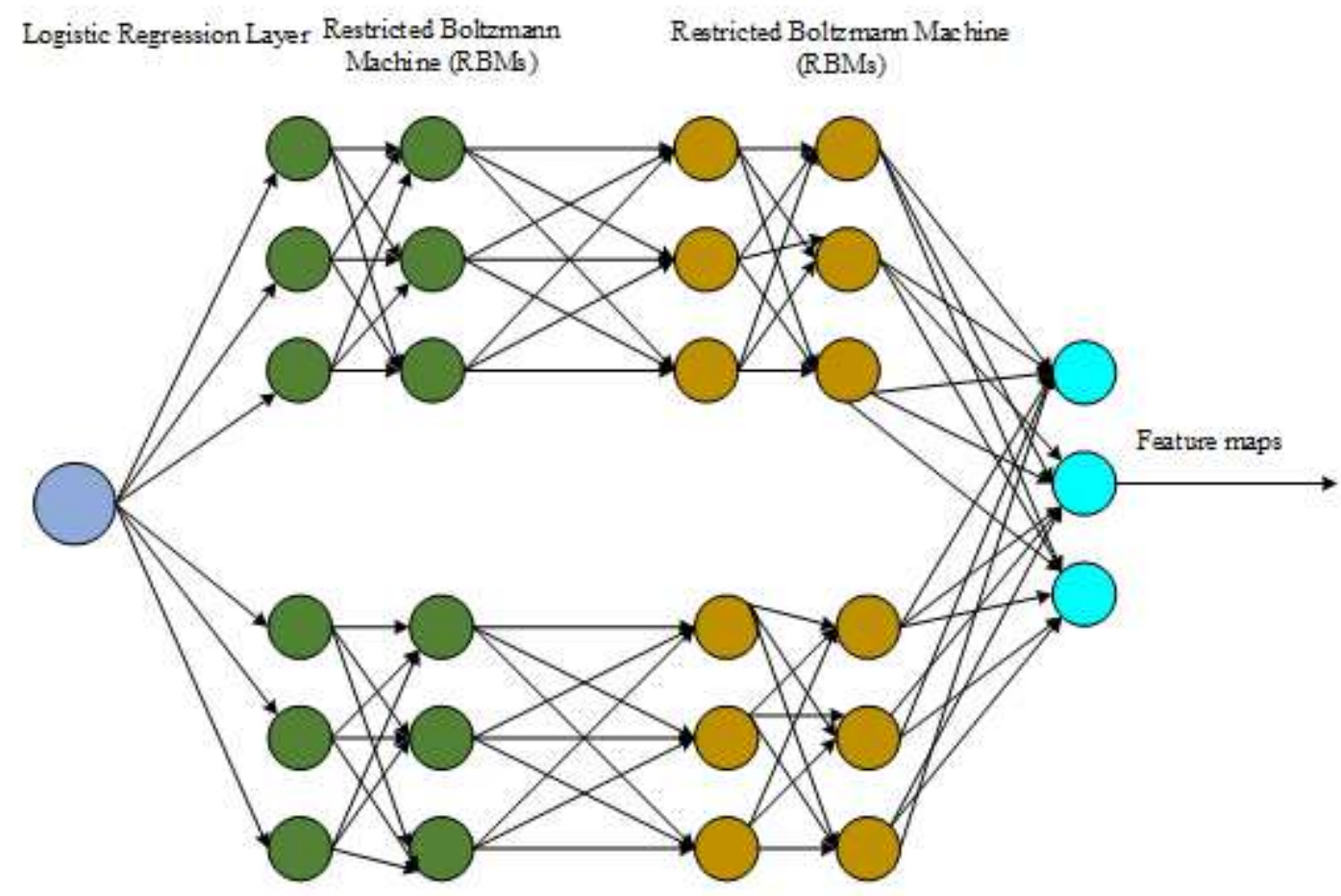

Figure. 2 Structure of Deep Belief Neural Network

An RPM is an energy-based production model in which I have parallel observable units (observed factors), where $\mathrm{v}=[v 1, v 2, \ldots, v I]$ where $v i \in\{0,1\}$, and $\mathrm{J}$ is a layer that Includes secret units (explanatory elements), where $h=[h 1, h 2, \ldots, h J]$ where $j \in\{0,1\}$, with bilateral weight associations. RBMs follow the encoderdecoder worldview. In this worldview, an encoder changes the contribution of an element vector representation from which a decoder can recreate the first information. In the case of RBMs, both coded imagery and (decoded) remaking are naturally normal. The encoder-encoder design is involved in shedding light on: (i) Following the production, the element vector can be processed in an auxiliary way, and (ii) the model can analyze how well it captures significant data by recreating the information. Given the observed express, the energy of the combined design of the transparent and closed units $(v, h)$ is provided by the condition underlying,

$$
\begin{aligned}
& E(v, h)=-C V^{T}-b h^{T}-h W v^{T} \\
& =-\sum_{i=1}^{I} C_{i} V_{i}-\sum_{j=1}^{j} b_{j} h_{j}-\sum_{j=1}^{J} \sum_{i=1}^{I} W_{j i} v_{i} h_{j}
\end{aligned}
$$

Where $W \in I R^{J \times I}$ is a lathe with RBM association loads, $c=[c 1, c 2, \ldots, c I] \in I R^{I}$ is the forerunner of observable units and $b=[b 1, b 2, \ldots, b J] \in I R^{J}$ The inclination of secret units. To break the equilibrium, loads are usually introduced with small irregular properties (for example somewhere in the range of .00 .01 and 0.01 ). The 
secret slope can introduce $b_{j}$ with an enormous negative value (e.g. -4$)$ to enhance porosity and significant units' dependence, $c i$, recording to $\log \left(\frac{p_{i}}{1-p_{i}}\right)$, where $p_{i}$ is the number of vectors produced in which vi $=1$. The learning method needs to be changed (in early productions) given that the noticeable unit is likely to have been activated i, so gradually merges with $p_{i}$. RBM expresses an opportunity for each system $(v, h)$, which is depicted in the underlying condition,

$$
p(v, h)=\frac{e^{-E(v, h)}}{Z}
$$

Where $Z$ is a standardization constant called parcel work in solidarity with the actual structure, which is obtained by summarizing the energy of all imaginary $(v, h)$ systems.

$$
z=\sum_{v, h} e^{-E(v, h)}
$$

Since there is no correlation between any two units within the same layer, given that a certain irregular information configuration is $\mathrm{v}$, each secret unit is free from each other, and $h$ is the probability of a given $v$,

$$
p(h \mid v)=\prod_{j} p\left(h_{j}=1 \mid v\right)
$$

Where,

$$
p\left(h_{j}=1 \mid v\right)=\sigma\left(b_{j}+\sum_{i=1}^{I} v_{i} w_{j i}\right)
$$

For execution purposes, $h_{j}$ is set to 1 when $p\left(h_{j}=1 \mid v\right)$ is more important than the given random number (assigned somewhere in the range 0 and 1 ) and in any case 0 . In addition to a certain secret level, the probability of $h$ given $v$ is given,

$$
p(v \mid h)=\prod_{i} p\left(v_{i}=1 \mid h\right)
$$

Where,

$$
p\left(v_{i}=1 \mid h\right)=\sigma\left(c_{i}+\sum_{j=1}^{j} h_{j} w_{j i}\right)
$$

When using the information vector to reproduce (18), it is necessary to double the confidentiality levels. The use of real probabilities would abuse the data barrier, which goes into a rigid order and is forced to bypass everything that treats secret units as little data. Given the external potential sent to the apparent vector, $V$,

$$
p(v)=\sum_{h} p(v, h)=\frac{1}{z} \sum_{h} e^{-E(v, h)}
$$

Then, if a specific product vector is given, its change can be elevated by changing $t$.

\subsection{Chimp Optimization Algorithm}


In this proposed methodology, COA is utilized to select the optimal DBNN training parameters. The mathematical formulation of COA is presented as follows,

\subsubsection{Inspiration}

Normally, the fission-fusion society is a chimp's society. This is considered as one of the societies, the combination of society may be a time-variant function. Additionally, in society, each member has a specific duty and special ability that may change over time. From the consideration, the aim of independent concepts is developed in this algorithm. Hence, every group of chimpanzees separately attempts to find the search space with its singular characteristics intended aimed at specific duty. Generally, four types of chimps are presented such as attackers, chasers, barriers, and drivers. Based on these types, the behaviors of the chimps in the hunting process are changed for efficient hunt operation. In the chimp's algorithm, the drivers have collected the prey without doing the hunting process. Barriers residence themselves in plants to create a dam crossway the leakage route of the prey [26]. The prey is grabbed by chasers rapidly. At last, the attackers are identifying the escape route of the prey down into the inferior canopy. Attackers are required to have more efficient in identifying the proceeding change of prey. Moreover, the attackers have been collected with the meat larger piece after an efficient hunt.

In Chimp calculation, the attack method is strictly related to actual ability, intelligence, and age. Also, Sims can change practices during a particular chase or interact with their strategy as a whole. It is authorized by the

chimps Chase to execute meat in exchange for social honors such as preparation and firm assistance. Henceforth, by opening another domain of interest and benefits. chimp may indirectly affect the chase. People use social motivation as chimps. In this way, the chimps have an advantage compared to other social predators. In addition to sexual motivation, start the sims to act turbulent as the last advance of the chase. Therefore, bulk chips drop the mistakes of obtaining meat independently. From the thinking of the social behavior of the Sims, it can be isolated into two primary stages, such as investigation and misuse. There is a way to track, prevent and drive prey in the investigation. Misuse is considered a prey attack. Details of misuse and investigation numbers are introduced as follows,

\section{Driving and chasing the prey}

In the $\mathrm{COA}$, the prey can be hunted throughout the exploitation also exploration stages. The mathematical design of chasing also driving prey is formulated as follows,

$$
\begin{aligned}
& D=\mid \text { c. } x^{\text {prey }}(T)-M \cdot x^{\text {chimp }}(T) \mid \\
& x^{\operatorname{chimp}}(T+1)=x^{\text {prey }}(T)-A \cdot D
\end{aligned}
$$


Where, $x^{\text {prey }}, x^{\text {chimp }}$ can be described as position vector of chimp and prey, $T$ can be described as a number of current iterations, and $A, M$ and $C$ can be described as coefficient vectors. The position vectors of the COA is computed based on the below equation,

$$
\begin{aligned}
& A=2 \cdot F \cdot R^{1}-a \\
& C=2 \cdot R^{2} \\
& M=\text { Chaotic Value }
\end{aligned}
$$

Where, $R^{1}$ and $R^{2}$ can be described as random parameters which in the variety of [0,1], $F$ can be described as coefficient which decreased non-linearly from 2.5 to 0 by the iteration procedure (in both explorations also exploitation). $M$ can be described as a chaotic parameter computed based on different chaotic maps. Hence, the vector describes the behaviour of sexual incentive of chimps in shooting behaviour.

\section{Fitness Evaluation}

Once the initial population is completed, the fitness function is computed. In the proposed methodology, random DBNN weight parameters are initialized. Based on the fitness function, the optimal weighting parameter of DBNN is computed. The fitness function is evaluated with the consideration of the PSNR value. The PSNR should be maximized to enable efficient secure operation. Hence, the fitness function is formulated with the maximization of PSNR. The fitness function is achieved by selecting the optimal weighting parameter value. The fitness function is mathematically formulated as follows,

$$
\begin{aligned}
& \mathrm{FF}=\mathrm{MAX}\{\mathrm{PSNR}\} \\
& P S N R=10 \log _{10}\left(\frac{M A X^{P}}{M S E}\right) \\
& M S E=\frac{1}{N * M} \sum_{X=1}^{N} \sum_{Y=1}^{M}\left[I_{\text {image }}(A, B)-I_{\text {d-image }}(A, B)\right]^{2}
\end{aligned}
$$

Where, $I_{\text {d-image }}(A, B)$ is described as identified images and $I_{\text {image }}(A, B)$ is described as an input image. Based on the fitness function, the weight parameters are selected which are utilized to enhance the optimal classification procedure. A complete description of the vector value is explained in the next section.

\section{Exploration phase}

The attack behaviour of the chimp's mathematical model is designed as follows, firstly, the chimps can provide the location of the prey, and secondly, they can orbit it. Finally, predators are generally maintained by attackers. The chaser, barrier, and driver are usually involved in the hunt. At the research stage, there was no 
information about the optimal condition of the prey during the initial repetition. This state of the chaser, block and drive must be updated using the attacker's status. So, four optimal solutions can be saved and the other chimps are stopped to update the positions related to the locations of the best chimps. This creation is presented mathematically as follows,

$$
\begin{aligned}
& d^{\text {Attacker }}=\left|C^{1} X^{\text {attacker }}-M^{1} D\right| \\
& d^{\text {Barrier }}=\left|C^{2} X^{\text {barrier }}-M^{2} X\right| \\
& d^{\text {Chaser }}=\left|C^{3} X^{\text {Chaser }}-M^{3} X\right| \\
& d^{\text {Driver }}=\left|C^{4} X^{\text {driver }}-M^{4} X\right| \\
& X^{1}=X^{\text {Attacker }}-A^{1}\left(d^{\text {Attacker }}\right) \\
& X^{2}=X^{\text {Barrier }}-A^{2}\left(d^{\text {Barrier }}\right) \\
& X^{3}=X^{\text {Chaser }}-A^{3}\left(d^{\text {Chaser }}\right) \\
& X^{4}=X^{\text {Driver }}-A^{4}\left(d^{\text {Driver }}\right) \\
& X(T+1)=\frac{X^{1}+X^{2}+X^{3}+X^{4}}{4}
\end{aligned}
$$

The search agent position is updated in the search space based on another chimp position. so, the chimp's final position is arbitrarily placed in the orbit which is described as drivers, chasers, barrier, and attacker positions.

\section{Exploitation phase}

As beforehand mentioned, the chimps will hunt the victim by attacking process while the prey stops running. In the attacking process of chimps, the value of $\mathrm{f}$ is linearly minimized. The vector of $a$ also reduced in the manner of $f$ vector. Additionally, the $a$ is an arbitrary variable in the interval of $[-2 f, 2 f]$. Additionally, COA chasing, blocking, and driving mechanisms have reinforced exploration capability and it may still be at the risk of local minima trapping conditions. Hence, the exploration is a required portion to achieve the best results. In COA, chimps deviate to attack the prey and converge to attack the prey. The vector $a$ is located to mathematical design this characteristic so that inequality parameters. To avoid local optima entrapment, the chimps forced to diverge from prey which formulated as $|a|>1$. To achieve global optima, the chimps forced to converge at prey location which formulated as $|a|<1$.

\section{Exploitation phase using the social incentive}


In the COA, the social incentive and society of chimps which related to meat hunting. In the final stage of the chimp hunting process, the chimp may abort its hunting process. Hence, they chaotically attempt to grab hunting meat for social essences. These characteristics of the chaotic map are designed with chaotic maps which formulated as follows,

$$
X^{\operatorname{chimp}}(T+1)=\left\{\begin{array}{lll}
x^{\text {prey }}(t)-A . D & \text { if } & \mu<0.5 \\
\text { Chaotic value } & \text { if } & \mu>0.5
\end{array}\right.
$$

Where $\mu$ can be described as an arbitrary number in the interval of $[0,1]$. Initially, they generate a random population of chimps. Secondly, all chimps are arbitrarily divided by different groups such as driver, chaser, barrier, also attacker. After that, every chimp position updates the f coefficients with the consideration of the own group method. The optimal prey location is identified in the iteration based on driver, chaser, barrier, and attacker. Then the distance from the prey, the positions are updated [27]. Additionally, the optimal tuning of the $m$ and $c$, the fast convergence rate and faster. Additionally, the value of $f$ can be adjusted from 2.5 to 0 which empowers the process of exploitation. Finally, the condition of the divergence and iterations are checked which provides the optimal results to manage the results. With the help of COA, the optimal weighting parameter is selected. After that, the proposed classifier is utilized to classify breast cancer from mammogram images.

\section{Performance Evaluation}

The performance of the proposed methodology is evaluated and justified in this section. The proposed methodology is validated with the consideration of statistical measurements such as accuracy, sensitivity, specificity, ROC, AUC, and F_Measure respectively. The proposed methodology is compared with existing methods such as RF, SVM, and ANN respectively. The proposed method can be implemented with the consideration of the collected database of the Digital Database of Screening Mammography [28]. This DDSM is an open-source database of mammography images with different conditions such as normal and benign. This collected dataset is divided into two sets such as training set and testing set. From the collected database, $80 \%$ of the dataset is selected for the training phase and $20 \%$ of the dataset is selected for the testing phase. The implementation parameters of the proposed method are presented in table 1. The proposed method is validated by statistical measurements. The performance metrics of the statistical measurements are presented below in this section. The sample databases are illustrated in figure 3. 


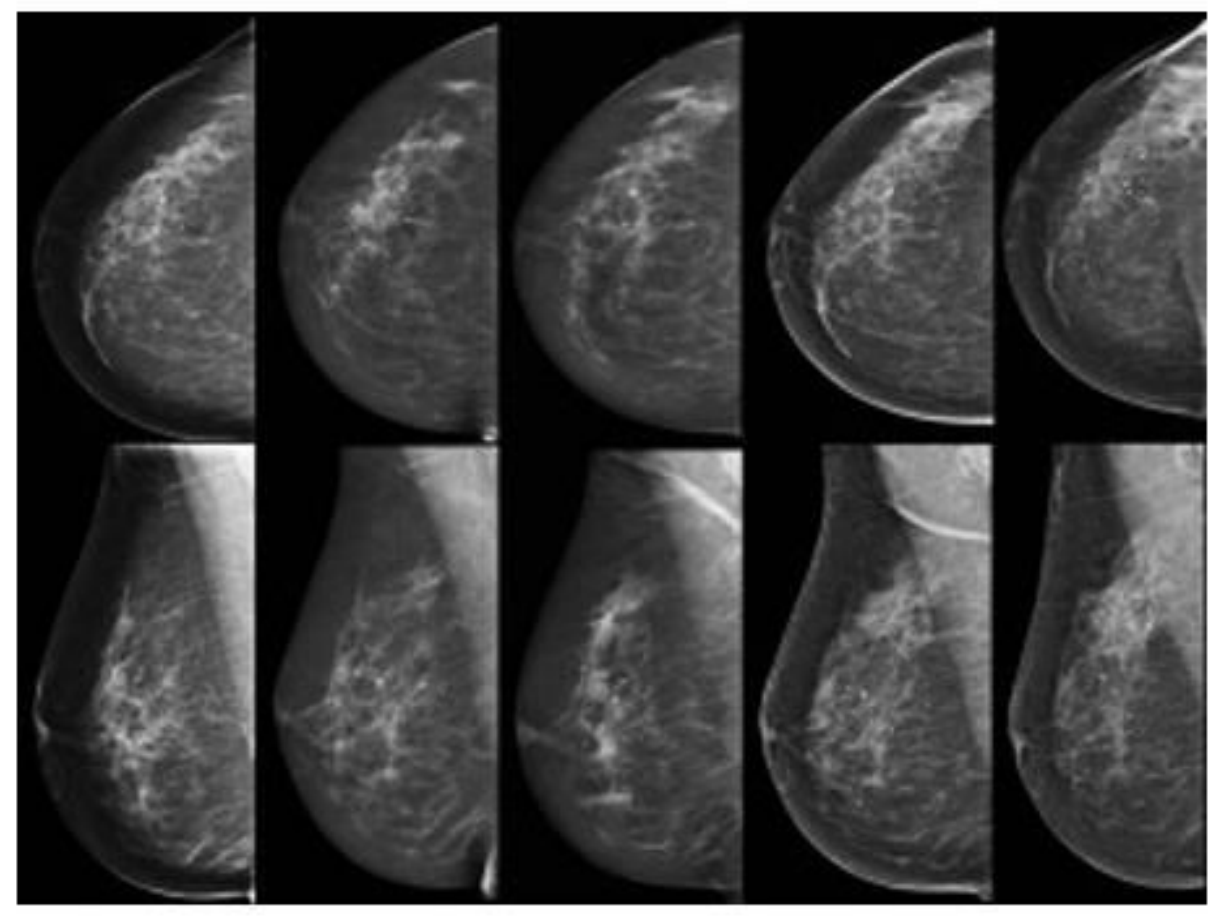

Figure. 3 Analysis of Sample database

Table. 1 Implementation parameters of the proposed methodology

\begin{tabular}{|c|c|c|c|}
\hline S. No & Method & Description & Value \\
\hline 1 & \multirow{9}{*}{ Proposed method } & Inertia factor & 0.7298 \\
\hline 2 & & Maximum iteration & 100 \\
\hline 3 & & Initial population & 50 \\
\hline 4 & & Momentum & 0.9 \\
\hline 5 & & Learn rate drop period & 5 \\
\hline 6 & & Learn rate drop factor & 0.2 \\
\hline 7 & & Initial learn rate & 0.05 \\
\hline 8 & & Max Epochs & 15 \\
\hline 9 & & Minimum batch size & 500 \\
\hline
\end{tabular}

The proposed classifier is utilized to classify breast cancer from mammogram images. Breast cancer is presented during the testing process, then breast cancer is classified. If breast cancer is not presented during the testing process, then breast cancer is not classified. The confusion matrix is computed based on the following constraints,

* Breast cancer is presented and detected as presented scenario which is named as True Positive (TP).

- Breast cancer is not presented and detected as not presented which is named as True Negative (TN).

* Breast cancer is not presented but detected which is named as False Positive (FP).

* Breast cancer is presented and detected as not presented which is named as False Negative (FN). 
Based on the progress of confusion matrix terms, the proposed methodology is evaluated by performance metrics which are formulated as follows,

Accuracy: It is defined as a number of correctly detected data instances from the total number of instances.

The formula of accuracy is presented as follows,

$$
\text { Accuracy }=\frac{T N+T P}{T N+F P+T P+F N}
$$

Sensitivity: It is defined as the fraction of positive detected which correct. The precision effectively computed a complete probability and performance measure. The recall formulation presented as follows,

$$
\text { Precision }=\frac{T P}{T P+F P}
$$

Specificity: It can be defined as the ratio of correctly detected negative instances to total negative instances which formulated as follows,

$$
\text { specificity }=\frac{T N}{(T N+F P)}
$$

F_Measure: The F_Measure must be presented among 0 to 1 . The worst value is 0 and the best value is 1 . The F_Measure can be represented as follows,

$$
F_{\text {Measure }}=\frac{2 T P}{(2 T P+F P+F N)}
$$

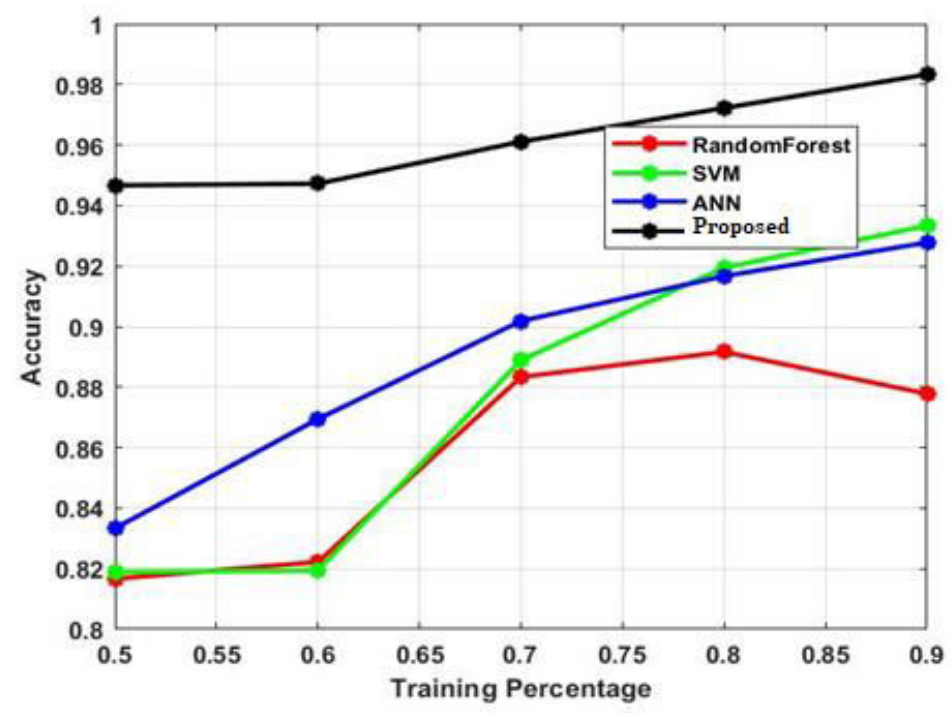

Figure. 4 Analysis of Accuracy 


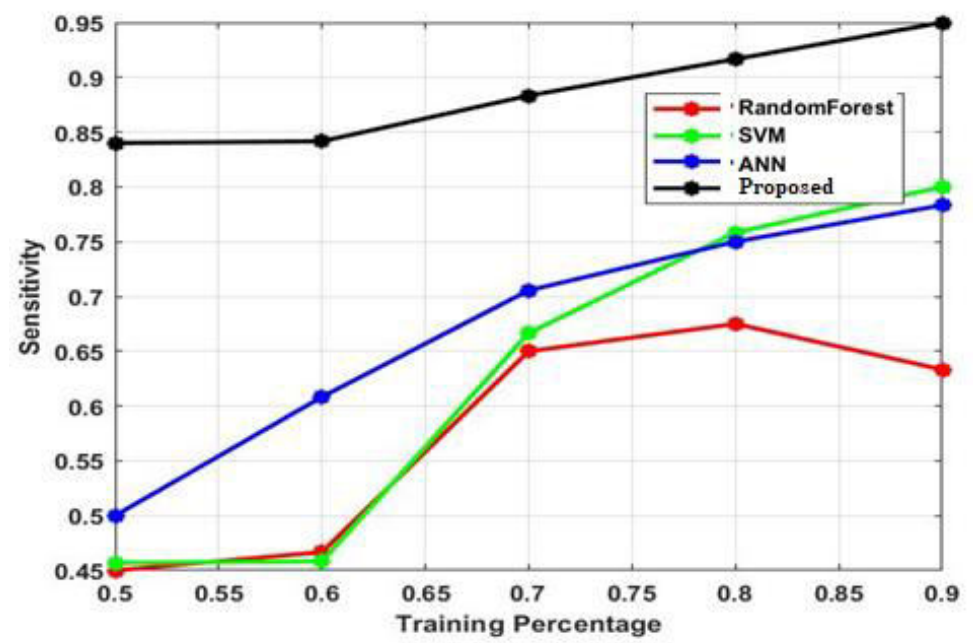

Figure. 5 Analysis of Sensitivity

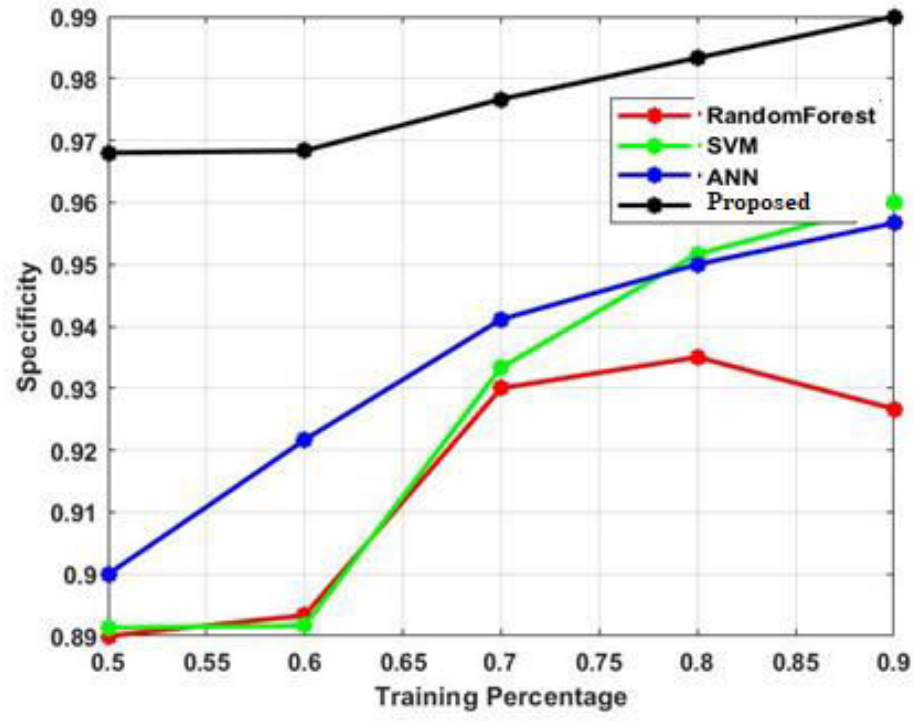

Figure. 6 Analysis of Specificity 


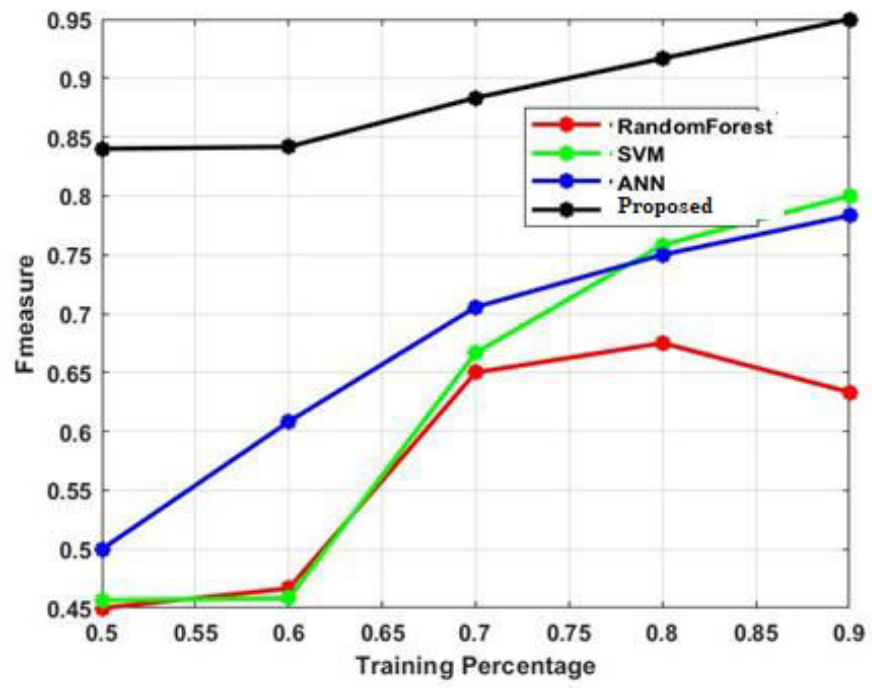

Figure. 7 Analysis of F_Measure

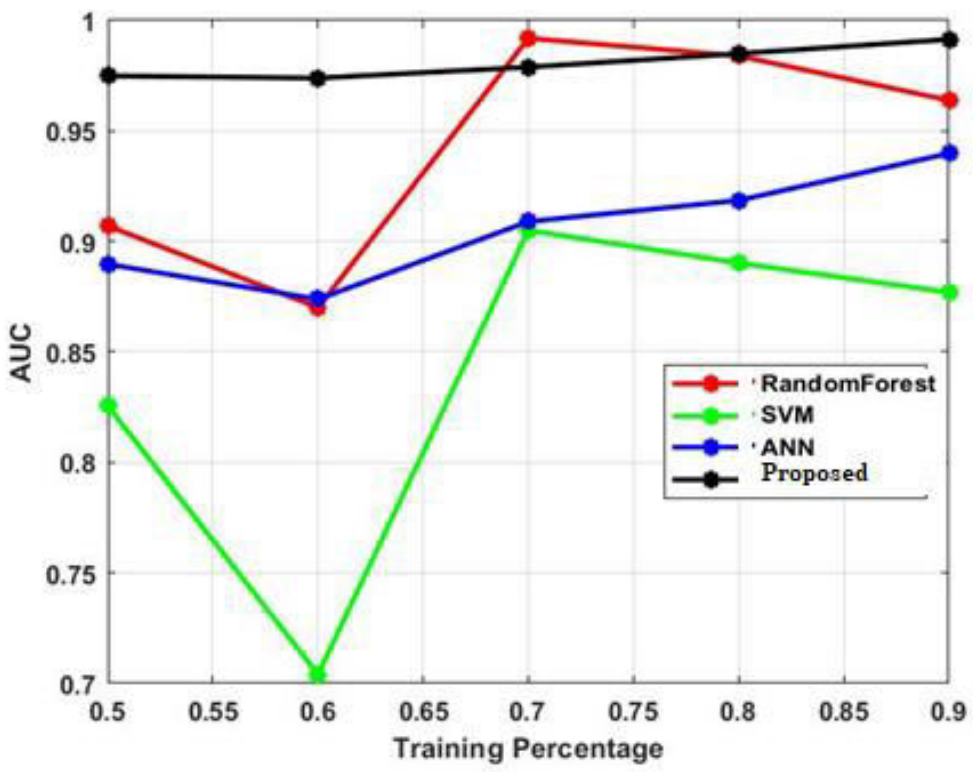

Figure. 8 Analysis of AUC

The proposed method is validated with the consideration of the comparison analysis. The proposed method is compared with the existing methods such as RF, SVM, and ANN respectively. The proposed methodology accuracy is illustrated in figure 4 . The proposed methodology has been achieved a 0.95 accuracy. Similarly, the existing methods of RF, SVM, and ANN have been achieved the accuracy of $0.82,0.81$, and 0.83 respectively. The AUC analysis also depicted in the figure 8. From the comparison analysis, the proposed methodology has been 
achieved the best results in terms of accuracy. The proposed method is validated with the consideration of the comparison analysis. The proposed method is compared with the existing methods such as RF, SVM, and ANN respectively. The proposed methodology sensitivity is illustrated in figure 5. The proposed methodology has been achieved the 0.85 sensitivity. Similarly, the existing methods of RF, SVM, and ANN have been achieved the sensitivity of $0.46,0.45$, and 0.50 respectively. From the comparison analysis, the proposed methodology has been achieved the best results in terms of sensitivity.

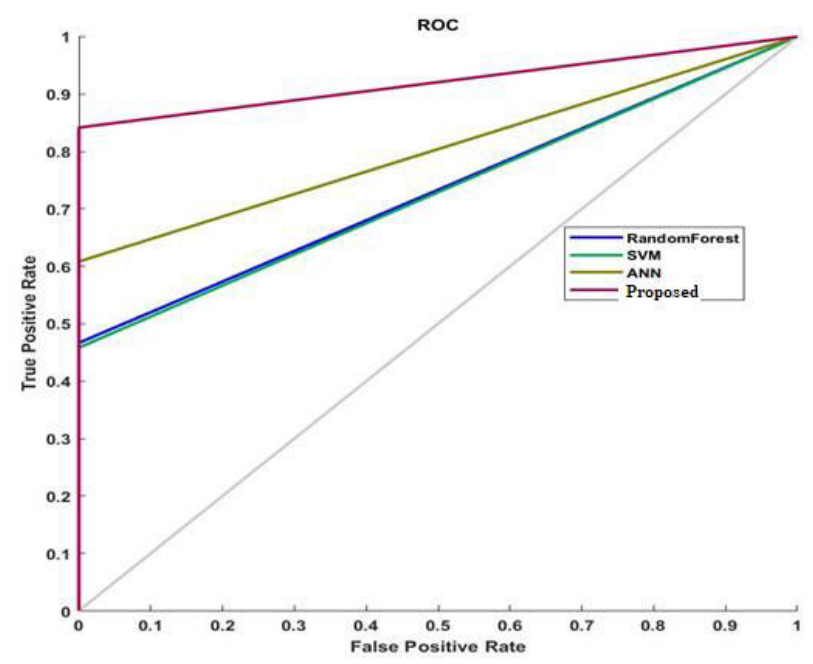

(a)

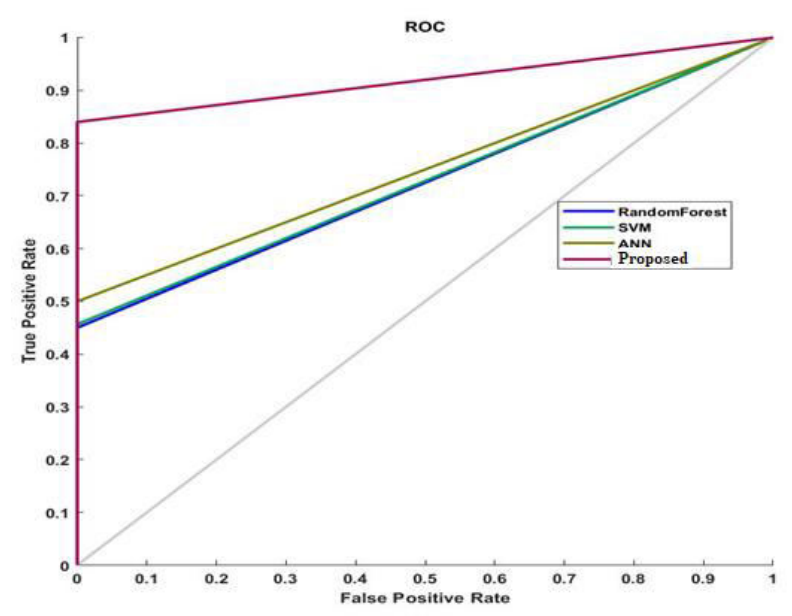

(b)

Figure. 9 Analysis of ROC (a) $40 \%$ training and (b) $50 \%$ training

The proposed method is validated with the consideration of the comparison analysis. The proposed method is compared with the existing methods such as RF, SVM, and ANN respectively. The proposed methodology 
specificity is illustrated in figure 6 . The proposed methodology has been achieved the 0.97 specificity. Similarly, the existing methods of RF, SVM, and ANN have been achieved the specificity of $0.89,0.88$, and 0.9 respectively. From the comparison analysis, the proposed methodology has been achieved the best results in terms of specificity. The proposed method is validated with the consideration of the comparison analysis. The proposed method is compared with the existing methods such as RF, SVM, and ANN respectively. The proposed methodology F_Measure is illustrated in figure 7. The proposed methodology has been achieved the 0.85 F_Measure. Similarly, the existing methods of RF, SVM, and ANN have been achieved the F_Measure of $0.5,0.45$, and 0.46 respectively. From the comparison analysis, the proposed methodology has been achieved the best results in terms of F_Measure. The ROC characteristics of the proposed methodology are analysed with the different training sets such as $10 \%$ of training, $20 \%$ of training, $30 \%$ of training, $40 \%$ of training, and $50 \%$ of training which are illustrated in figure 9 . From the analysis, the proposed methodology has been achieved the best results in terms of ROC.

\section{Conclusion}

An automated technique has been utilized in the mammogram images according to their micro-calcification classification. The automated technique has been working with the combination of DBNN and COA. The proposed method has been working with three phases such as pre-processing phase, feature extraction, and classification phase. In the pre-processing phase, a median filter has been utilized to remove unwanted information from the images. In the feature extraction phase, GLCM, SIFT, and Hu moments have been utilized to extract essential features from mammogram images. After that, the detection and classification have been performed on the mammogram images according to their micro-calcifications with the utilization of the proposed advanced deep learning method. From the classification stage, the normal and abnormal images are identified from the images. The proposed method has been implemented in the MATLAB platform and analysed their statistical performances like accuracy, sensitivity, specificity, precision, recall, and F-measure. To evaluate the effectiveness of the proposed method this has been compared with the existing method such as SVM, RF, and ANN. From the analysis, the proposed methodology has been achieved the best results in breast tumor classification.

\section{Data Availability Statement}

Not Applicable 


\section{Funding}

Not Applicable

\section{Conflicts of interest}

Not Applicable

\section{Availability of data and material}

Not Applicable

\section{Code availability}

Not Applicable

\section{References}

[1]. Hiba Asri, Hajar Mousannif, Hassan Al Moatassime, and Thomas Noel, "Using machine learning algorithms for breast cancer risk prediction and diagnosis", Procedia Computer Science, (2016) 83: 1064-1069, DOI: 10.1016/j.procs.2016.04.224

[2]. Md Milon Islam, Md Rezwanul Haque, Hasib Iqbal, Md Munirul Hasan, Mahmudul Hasan and Muhammad Nomani Kabir, "Breast cancer prediction: a comparative study using machine learning techniques", SN Computer Science, (2020) 1(5):1-14, DOI: 10.1007/s42979-020-00305-w

[3]. Mogana Darshini Ganggayah, Nur Aishah Taib, Yip Cheng Har, Pietro Lio and Sarinder Kaur Dhillon, "Predicting factors for survival of breast cancer patients using machine learning techniques", BMC Medical Informatics and Decision Making (2019) 19(1):1-17, DOI: 10.1186/s12911-019-0801-4

[4]. Puja Gupta and Shruti Garg, "Breast cancer prediction using varying parameters of machine learning models", Procedia Computer Science, (2020) 171:593-601, DOI: 10.1016/j.procs.2020.04.064

[5]. Santhosh Kumar. B, Daniya. T and Ajayan. J, "Breast cancer prediction using machine learning algorithms", International Journal of Advanced Science and Technology, (2020) 29(3):7819-7828.

[6]. Min-Wei Huang, Chih-Wen Chen, Wei-Chao Lin, Shih-Wen Ke and Chih-Fong Tsai, "SVM and SVM ensembles in breast cancer prediction", PLoS ONE, (2017) 12(1):1-14, DOI: 10.1371/journal.pone.0161501

[7]. Leili Tapak, Nasrin Shirmohammadi-Khorram, Payam Amini, Behnaz Alafchi, Omid Hamidi and Jalal Poorolajal, "Prediction of survival and metastasis in breast cancer patients using machine learning classifiers", Clinical Epidemiology and Global Health, (2019) 7(3):293-299, DOI: 10.1016/j.cegh.2018.10.003 
[8]. Mandeep Rana, Pooja Chandorkar, Alishiba Dsouza and Nikahat Kazi, "Breast cancer diagnosis and recurrence prediction using machine learning techniques", International Journal of Research in Engineering and Technology, (2015) 4(4):372-376, DOI: 10.15623/ijret.2015.0404066

[9]. Wenbin Yue, Zidong Wang, Hongwei Chen, Annette Payne and Xiaohui Liu, "Machine learning with applications in breast cancer diagnosis and prognosis", Designs, (2018) 2(2):13, DOI: 10.3390/designs2020013

[10]. Habib Dhahri, Eslam Al Maghayreh, Awais Mahmood, Wail Elkilani and Mohammed Faisal Nagi, "Automated breast cancer diagnosis based on machine learning algorithms", Journal of Healthcare Engineering, (2019) 2019:1-11, DOI: 10.1155/2019/4253641

[11]. Wenqing Sun, Tzu-Liang Bill Tseng, Jianying Zhang and Wei Qian, "Enhancing deep convolutional neural network scheme for breast cancer diagnosis with unlabeled data", Computerized Medical Imaging and Graphics, (2017) 57:4-9, DOI: 10.1016/j.compmedimag.2016.07.004

[12]. Rahimeh Rouhi, Mehdi Jafari, Shohreh Kasaei and Peiman Keshavarzian, "Benign and malignant breast tumors classification based on region growing and CNN segmentation", Expert Systems with Applications, (2015) 42(3):990-1002, DOI: 10.1016/j.eswa.2014.09.020

[13]. Zhiqiong Wang, Mo Li, Huaxia Wang, Hanyu Jiang, Yudong Yao, Hao Zhang and Junchang Xin, "Breast cancer detection using extreme learning machine based on feature fusion with CNN deep features", IEEE Access, (2019) 7:105146-105158, DOI: 10.1109/ACCESS.2019.2892795

[14]. Juan Zuluaga-Gomez, Zeina Al Masry, Khaled Benaggoune, Safa Meraghni and Nourredine Zerhouni, "A CNN-based methodology for breast cancer diagnosis using thermal images", Computer Methods in Biomechanics and Biomedical Engineering: Imaging and Visualization, (2021) 9(2):131-145, DOI: $10.1080 / 21681163.2020 .1824685$

[15]. Lazaros Tsochatzidis, Panagiota Koutla, Lena Costaridou and Ioannis Pratikakis, "Integrating segmentation information into CNN for breast cancer diagnosis of mammographic masses", Computer Methods and Programs in Biomedicine, (2021) 200:105913, DOI: 10.1016/j.cmpb.2020.105913

[16]. Naveed Chouhan, Asifullah Khan, Jehan Zeb Shah, Mazhar Hussnain and Muhammad Waleed Khan, "Deep convolutional neural network and emotional learning based breast cancer detection using digital mammography", Computers in Biology and Medicine (2021) 132:104318, DOI: 10.1016/j.compbiomed.2021.104318 
[17]. Hua Li, Shasha Zhuang, Deng-ao Li, Jumin Zhao and Yanyun Ma, "Benign and malignant classification of mammogram images based on deep learning", Biomedical Signal Processing and Control, (2019) 51:347-354, DOI: $10.1016 /$ j.bspc.2019.02.017

[18]. Agnes. S, Anitha. J, Pandian. S and Peter. J, "Classification of mammogram images using multiscale all convolutional neural network (MA-CNN)", Journal of Medical Systems, (2020) 44(1):1-9, DOI: $10.1007 / \mathrm{s} 10916-019-1494-\mathrm{Z}$

[19]. Dina A. Ragab, Omneya Attallah, Maha Sharkas, Jinchang Ren, and Stephen Marshall, "A framework for breast cancer classification using multi-DCNNs", Computers in Biology and Medicine (2021) 131:104245, DOI: 10.1016/j.compbiomed.2021.104245

[20]. Raghavendra.U, Rajendra Acharya. U, Hamido Fujita, Anjan Gudigar, Jen Hong Tan and Shreesha Chokkadi, "Application of Gabor wavelet and Locality Sensitive Discriminant Analysis for automated identification of breast cancer using digitized mammogram images", Applied Soft Computing Journal, (2016) 46:151-161, DOI: 10.1016/j.asoc.2016.04.036

[21]. Langarizadeh. M, Mahmud. R, Ramli. A, Napis. S, Beikzadeh. M and Rahman. W, "Improvement of digital mammogram images using histogram equalization, histogram stretching and median filter", Journal of Medical Engineering and Technology, (2011) 35(2):103-108, DOI: 10.3109/03091902.2010.542271

[22]. Vibhav Prakash, Singh, Ayush Srivastava, Devang Kulshreshtha, Arpit Chaudhary and Rajeev Srivastava, "Mammogram classification using selected GLCM features and random forest classifier", International Journal of Computer Science and Information Security (IJCSIS), (2016) 14(6):82-87.

[23]. Taimoor Shakeel Sheikh, Yonghee Lee and Migyung Cho, "Histopathological classification of breast cancer images using a multi-scale input and multi-feature network", Cancers, (2020) 12(8):1-21, DOI: $10.3390 /$ cancers 12082031

[24]. Shallu Sharma and Rajesh Mehra, "Conventional machine learning and deep learning approach for multiclassification of breast cancer histopathology images - a comparative insight", Journal of Digital Imaging, (2020) 33(3):632-654, DOI: 10.1007/s10278-019-00307-y

[25]. Ahmed M.Abdel-Zaher and Ayman M. Eldeib, "Breast cancer classification using deep belief networks", Expert Systems with Applications, (2016) 46:139-144, DOI: 10.1016/j.eswa.2015.10.015 
[26]. Khishe. M and Mohammad Reza Mosavi, "Chimp optimization algorithm", Expert Systems with Applications, (2020) 149:113338, DOI: 10.1016/j.eswa.2020.113338

[27]. Khishe. M and Mosavi. M, "Classification of underwater acoustical dataset using neural network trained by chimp optimization algorithm", Applied Acoustics, (2020) 157:107005, DOI: 10.1016/j.apacoust.2019.107005

[28]. https://www.kaggle.com/skooch/ddsm-mammography 